\title{
Diffuse alveoläre Hämorrhagie mit späterer ANCA-Positivität und Emphysembildung bei drei jungen Erwachsenen
}

\author{
Anna Stainer $^{\mathrm{a}} \quad$ Alex Rice $^{\mathrm{b}} \quad$ Anand Devaraj $^{\mathrm{c}} \quad$ Joseph Luke Barnett ${ }^{\mathrm{c}}$ \\ Jacqueline Donovan $^{d}$ Maria Kokosi ${ }^{\mathrm{a}}$ Andrew Gordon Nicholson ${ }^{\text {b, e }}$ \\ Tom Cairns $^{f} \quad$ Athol Umfrey Wells $^{a} \quad$ Elisabetta Augusta Renzoni ${ }^{a}$ \\ a Interstitial Lung Disease Unit, Royal Brompton Hospital, London, Großbritannien; \\ ${ }^{b}$ Department of Histopathology, Royal Brompton Hospital, London, Großbritannien; \\ 'Department of Radiology, Royal Brompton Hospital, London, Großbritannien; \\ ${ }^{\mathrm{d}}$ Department of Clinical Biochemistry, Royal Brompton Hospital, London, Großbritannien; \\ eNational Heart and Lung Institute, Imperial College London, London, Großbritannien; \\ ${ }^{f}$ Imperial College Healthcare NHS Trust, London, Großbritannien
}

\section{Schlüsselwörter}

ANCA · Pulmonale Hämosiderose · Hämoptysen .

Pulmonale Hämorrhagie · Pulmonale Vaskulitis · Emphysem .

ANCA-assoziierte Vaskulitis · AAV

\section{Zusammenfassung}

Hintergrund: Die diffuse alveoläre Hämorrhagie (DAH) ist durch die diffuse Anreicherung von roten Blutkörperchen in den Alveolen und durch Milchglastrübungen und/oder Konsolidierungen in der Computertomographie (CT) gekennzeichnet. Wenn keine nicht immunologischen Ursachen identifiziert werden können, wird die DAH klassischerweise in eine idiopathische DAH (idiopathische pulmonale Hämosiderose, $(\mathrm{PH})$ und eine autoimmun bedingte DAH eingeteilt. In der vorliegenden Arbeit beschreiben wir 3 Patienten mit zunächst als IPH eingestuften rezidivierenden pulmonalen Hämorrhagien, die Jahre nach der Erstmanifestation AntiMyeloperoxidase (MPO)-Antikörper, ein im CT sichtbares Emphysem und, in einem Fall, eine Nierenbeteiligung ausbildeten.

Fallbericht: Bei Patientin 1 wurde im Alter von 14 Jahren eine IPH diagnostiziert. Die Erkrankung war durch eine immunsuppressive Therapie nur schlecht beherrschbar, auch wenn ANCA über Jahre negativ blieben. Neunzehn Jahre nach der Erstmanifestation wurden bei der Patientin MPO-ANCA und eine leichte Nierenbeteiligung nachgewiesen. Die Patientin erhielt eine Behandlung mit Rituximab, auf die sie gut ansprach. Seit der Erstmanifestation waren im Thorax-CT durchgehend diffuse Milchglastrübungen und eine Verdickung von Interlobulärsepten erkennbar. Zehn Jahre später traten zystenartige Verschattungen auf, die mit einem Emphysem vereinbar waren und eine auffallende peribronchovaskuläre Verteilung zeigten. Bei Patient 2 wurde im Alter von 32 Jahren eine IPH diagnostiziert. Er erhielt Kortikosteroide und Methotrexat, worauf er wechselhaft ansprach. Elf Jahre nach der Erstmanifestation wurden MPO-ANCA nachgewiesen und im CT zeigte sich ein Emphysem mit peribronchovaskulärer Verteilung, das sich in der Folge wesentlich weiter ausdehnte. Bei Patient 3 wurde im Alter von 7 Jahren eine IPH diagnostiziert, die mit rezidivierenden Hämoptysen variablen Schweregrads einherging. Diese wurden intermittierend mit Kortikosteroiden behandelt, bis der Patient im Alter von 11 Jahren wegen schwerer DAH intubiert werden musste. Acht Jahre nach der Erstdiagnose wurden im CT emphysematöse Veränderungen beobachtet und 11 Jahre nach der Erstdiagnose wurden zum ersten Mal MPO-ANCA nachgewiesen.

Schlussfolgerungen: Unserer Ansicht nach zeigen diese 3 Fälle Folgendes: 1. die Möglichkeit, dass sich mehrere Jahre nach Erstma- 
nifestation einer DAH eine ANCA-Positivität ausbildet, 2. die Möglichkeit, dass eine DAH zu zystenartigen/emphysematösen Veränderungen mit peribronchovaskulärer Verteilung im CT führen kann. Darüber hinaus unterstreichen die Tatsache, dass die Patienten eine fortgesetzte immunsuppressive Therapie benötigten, und die Em- physembildung, dass Autoimmun-Phänomene eine Rolle spielen können, auch wenn die DAH zunächst als «idiopathisch» eingestuft wird. Es sind weitere Studien erforderlich, um die Beziehung zwischen DAH, ANCA-Positivität und Emphysembildung besser zu verstehen.

\section{Hintergrund}

Die diffuse alveoläre Hämorrhagie (DAH) ist durch eine intraalveoläre Anreicherung von roten Blutkörperchen mit diffusen Milchglastrübungen und/oder Konsolidierungen in der hochauflösenden Computertomographie (high-resolution computed tomography, HRCT) gekennzeichnet. Das klinische Bild der DAH reicht von asymptomatischen Patienten, bei denen die Diagnose als Zufallsbefund im Rahmen der bildgebenden Diagnostik und/ oder einer bronchoalveolären Lavage gestellt wird, bis hin zur lebensbedrohlichen akuten respiratorischen Insuffizienz. Histologisch ist die DAH durch hämosiderinbeladene Makrophagen, Fibrin-Ablagerungen, Typ-II-Pneumozytenhyperplasie, organisierende Pneumonie und akute Entzündungszustände gekennzeichnet. Kapillaritis ist, sofern vorhanden, mit einer interstitiellen Infiltration durch neutrophile Granulozyten und Zerstörung von Alveolenwänden assoziiert, wobei diese Veränderungen jedoch subtil und schwer zu identifizieren sein können [1].

Die Ätiologie der DAH ist breitgefächert und umfasst immunologische und nicht immunologische Ursachen. Zu den häufigsten immunologischen Ursachen der DAH gehören systemische Vaskulitiden und hier insbesondere Anti-Neutrophile cytoplasmatische Antikörper (ANCA)-assoziierte Vaskulitiden (AAV). Wenn keine Ursache oder Assoziation nachweisbar ist, wird die DAH als idiopathische pulmonale Hämosiderose (IPH) klassifiziert [2]. Wir beschreiben 3 Patienten mit rezidivierender pulmonaler Hämorrhagie, bei denen viele Jahre nach der Erstmanifestation Anti-Myeloperoxidase (MPO)-Antikörper auftraten und in der radiologischen Bildgebung zystenartige Bereiche sichtbar wurden, die an ein Emphysem denken ließen.

\section{Fallbericht}

\section{Patientin 1}

Die 14 Jahre alte junge Frau wurde mit Lethargie, Belastungsdyspnoe, mikrozytärer hypochromer Anämie, persistierendem Husten und multiplen Hämoptysen-Episoden in die Kinderlungenklinik am Royal Brompton Hospital (RBH) überwiesen. Die Lungenfunktion zeigte das Muster einer leichten restriktiven Ventilationsstörung (forcierte Vitalkapazität (forced vital capacity, FVC) 73\%, Einsekundenkapazität (forced expiratory volume per second, $\mathrm{FEV}_{1}$ ) 77,5\%, totale Lungenkapazität (total lung capacity, TLC) 77\%, Kohlenmonoxid-Transferfaktor (transfer factor for the lung for carbon monoxide, $\mathrm{T}_{\mathrm{LCO}}$ ) $85 \%$ und -Transferkoeffizient $\left.\left(\mathrm{K}_{\mathrm{CO}}\right) 110 \%\right]$. In der HRCT war eine ausgedehnte Milch- glastrübung über beiden Lungen zu sehen (Abb. 1a). Bei der bronchoalveolären Lavage (BAL) war die rückgewonnene Spülfüssigkeit zunehmend hämorrhagisch und es wurden zahlreiche hämosiderinbeladene Makrophagen identifiziert. Eine chirurgisch entnommene Lungenbiopsie ergab einen mit einer DAH vereinbaren Befund (Abb. 2). Wegen fehlender identifizierbarer Assoziationen wurde bei der Patientin eine IPH diagnostiziert und eine Behandlung mit Hydroxychloroquin eingeleitet. Allerdings blieb die Erkrankung unzureichend kontrolliert und es kam über die Jahre zu zahlreichen Schüben, wozu eine schlechte Compliance beigetragen haben kann.

Im Alter von 21 Jahren wurde die Patientin mit zunehmenden Atemproblemen, häufigen «infektiösen» Exazerbationen und täglichen leichten Hämoptysen in die Spezialabteilung für interstitielle Lungenerkrankungen (ILD) im Erwachsenenalter am $\mathrm{RBH}$ aufgenommen. Zu dem Zeitpunkt verneinte sie Symptome einer Kollagenose. Die Patientin hatte in den letzten 3 Jahren nur hin und wieder geraucht und kam insgesamt auf ein Packungsjahr. Das Autoimmun-Screening war negativ. Während in der indirekten Immunfluoreszenz (NOVA-lite, INOVA Diagnostics Inc., San Diego, USA) ein Färbungsmuster für atypische unspezifische antineutrophile zytoplasmatische Antikörper (ANCA) sichtbar war, fielen Tests auf Anti-MPO und Anti-Proteinase-3 (PR3) negativ aus (Fluoreszenz-Enzym-Immunassay mit dem Immunocap 250 Analysator (Thermo Scientific ImmunoDiagnostics, Milton Keynes, UK)). Im HRCT des Thorax waren erneut diffuse Milchglastrübungen und außerdem verdickte Interlobulärsepten zu erkennen (Abb. 1b). Die Lungenfunktionsprüfung zeigte nun eine leichte obstruktive Ventilationsstörung bei deutlich verschlechterter $\mathrm{T}_{\mathrm{LCO}}(48 \%)$, ohne Anhalt für eine pulmonale Hypertonie in der Echokardiographie. In den folgenden Jahren erhielt die Patienten orale und intravenöse Kortikosteroide in wechselnder Dosierung. Sie vertrug weder Azathioprin noch Methotrexat. Durch Mycophenolat-Mofetil konnten die Hämoptysen einige Jahre lang beherrscht werden, allerdings musste die Patientin weiter Prednisolon einnehmen, was zu einer deutlichen Gewichtszunahme führte. Als sie Ende 20 war, traten erneut Hämoptysen auf. Es wurde eine leichte Beeinträchtigung der Nierenfunktion mit mikroskopischer Hämaturie aber ohne Erythrozytenzylinder diagnostiziert.

Im Alter von 32 Jahren bildete die Patientin p-ANCA aus und Anti-MPO waren mit 3,5 U/ml positiv (bestätigt durch eine zweite Probe $(27 \mathrm{U} / \mathrm{ml}))$. Im HRCT war zusätzlich zu der bekannten Milchglastrübung als neuer Befund ein Emphysem erkennbar (Abb. 1c-d), dessen Ausbreitung entlang der Lungengefäße für
102

Kompass Pneumol 2020;8:101-108 DOI: $10.1159 / 000505468$ 
Abb. 1. Patientin 1. Radiologischer Verlauf. Axiale CT-Bilder der Lungen. (a) Im Alter von 14 Jahren (zum Zeitpunkt der Erstpräsentation) sind ausgedehnte Milchglasinfiltrate mit geographischer Konfiguration und scharfer Abgrenzung zwischen den Infiltraten und dem gesunden Lungengewebe zu sehen. Es ist keine Verdickung von Interlobulärsepten erkennbar. (b) Im Alter von 21 Jahren erscheinen die Milchglasinfiltrate diffuser und es ist eine gleichmäßige Verdickung von Interlobulärsepten zu erkennen (Pfeil). (c) Im Alter von 31 Jahren wird zusätzlich zu persistierenden Milchglasinfiltraten als neuer Befund ein Emphysem erkennbar. (d) Die Vergrößerung von (c) zeigt, dass sich das Emphysem entlang der Lungengefäße ausdehnt (Pfeil), was auf ein interstitielles Emphysem hinweist.

Abb. 2. Patientin 1. Histologischer Befunde einer Lungenbiopsie. (a) Deutliche Anreicherung von hämosiderinbeladenen Makrophagen in den Alveolen (H\&E x 40). (b) Leichte chronische Bronchiolitis mit kleinen lymphoiden Aggregaten (H\&E x 100). (c) Fokale alveoläre Fibrinakkumulation und leichte chronische interstitielle Entzündung (H\&E x 400). (d) Leichte chronische interstitielle Entzündung und reaktive Typ2-Pneumozytenhyperplasie (H\&E x 400).
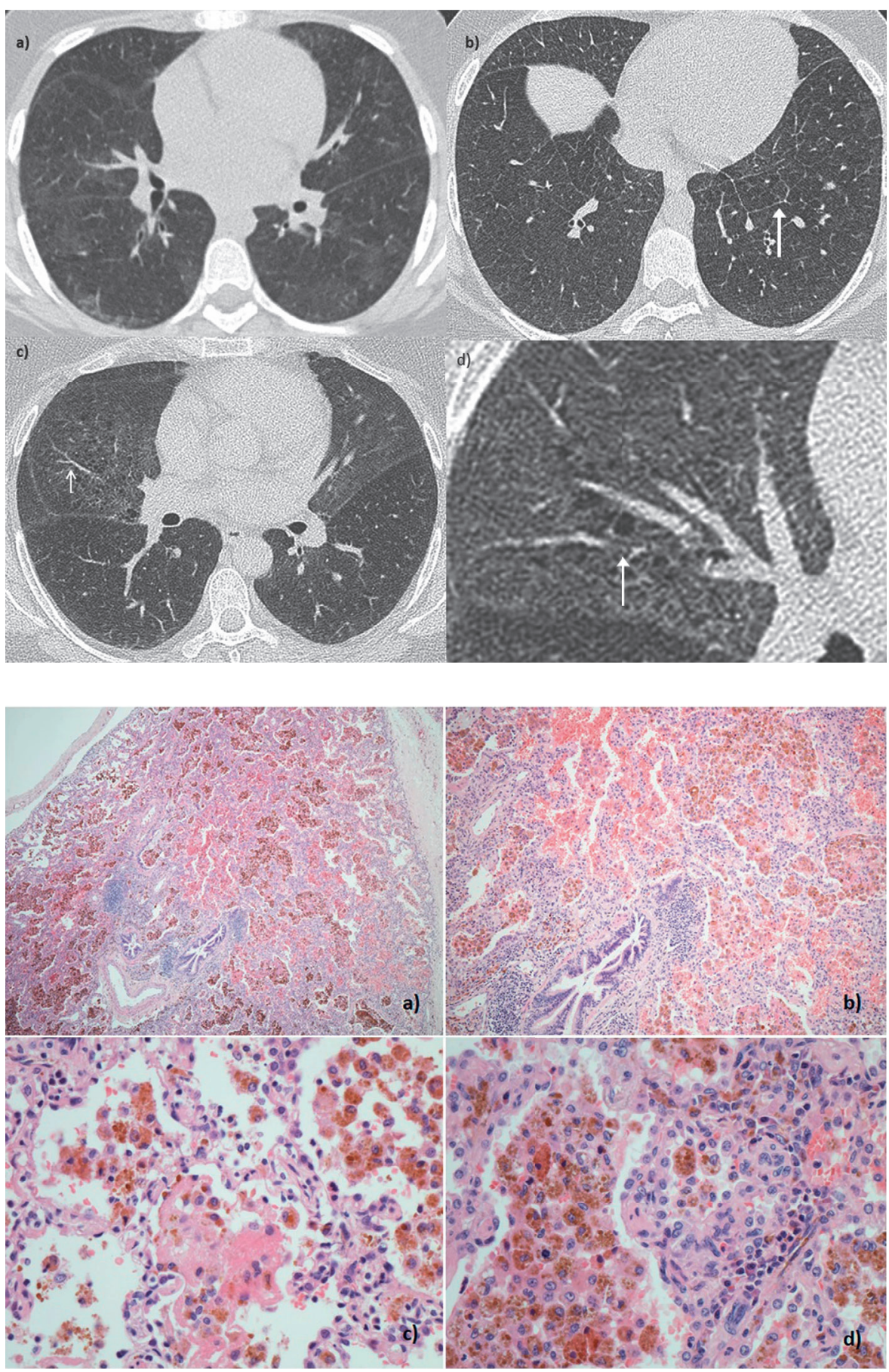

ein interstitielles Emphysem sprach. Es wurde eine Behandlung mit intravenösem Cyclophosphamid begonnen, auf die die Patientin partiell ansprach. Anschließend erhielt sie Rituximab, was in 6-monatlichen Intervallen fortgesetzt wurde. Bei der letzten Nachuntersuchung 10 Monate nach Beginn der Rituximab-Therapie berichtete die Patientin über eine Besserung ihrer Atemprobleme und beschrieb keine weiteren Hämoptysen. Die Nierenfunktion war leicht verändert, aber stabil, und die Lungenfunktion war stabil bei leichter obstruktiver Ventilationsstörung und überproportionaler Reduktion von $\mathrm{T}_{\mathrm{LCO}}$ und $\mathrm{K}_{\mathrm{CO}}$, was an eine Emphysementwicklung denken ließ.

\section{Patient 2}

Ein 34 alter Mann wurde mit seit 2 Jahren bestehender Belastungsdyspnoe sowie rezidivierenden Hämoptysen und transfusionspflichtiger Anämie in die ILD-Abteilung am RBH überwiesen. Im Rahmen der Erstuntersuchungen am lokalen Krankenhaus hatte ein HRCT des Thorax eine diffuse Milchglastrübung (Abb. 3a) gezeigt, während das Autoimmun-Screening negativ und die Lungenfunktionsprüfung ohne Befund waren und eine chirurgisch gewonnene Lungenbiopsie eine für DAH charakteristische Histologie aufwies (Abb. 4). Der Patient war Traktorfahrer in einem landwirtschaftlichen Betrieb und musste im Rah- 
Abb. 3. Patient 2. Radiologischer Verlauf. Axiale CT-Bilder der Lungen. (a) Das CT bei der Erstmanifestation (Alter von 34 Jahren) zeigt leichte Milchglasinfiltrate, eine regelmäßige Verdickung von Interlobulärsepten und ein subpleurales Knötchen im linken Unterlappen. (b) Im Alter von 40 Jahren imponieren die Milchglasinfiltrate deutlicher. (c) Im Alter von 42 Jahren sind ein perivaskuläres Emphysem/Zysten erkennbar (weißer Pfeil), die (d) 2 Jahre später zum Emphysem fortgeschritten sind. Wie auch in Abb. 1 zeigt das Emphysem eine perivaskuläre Verteilung.

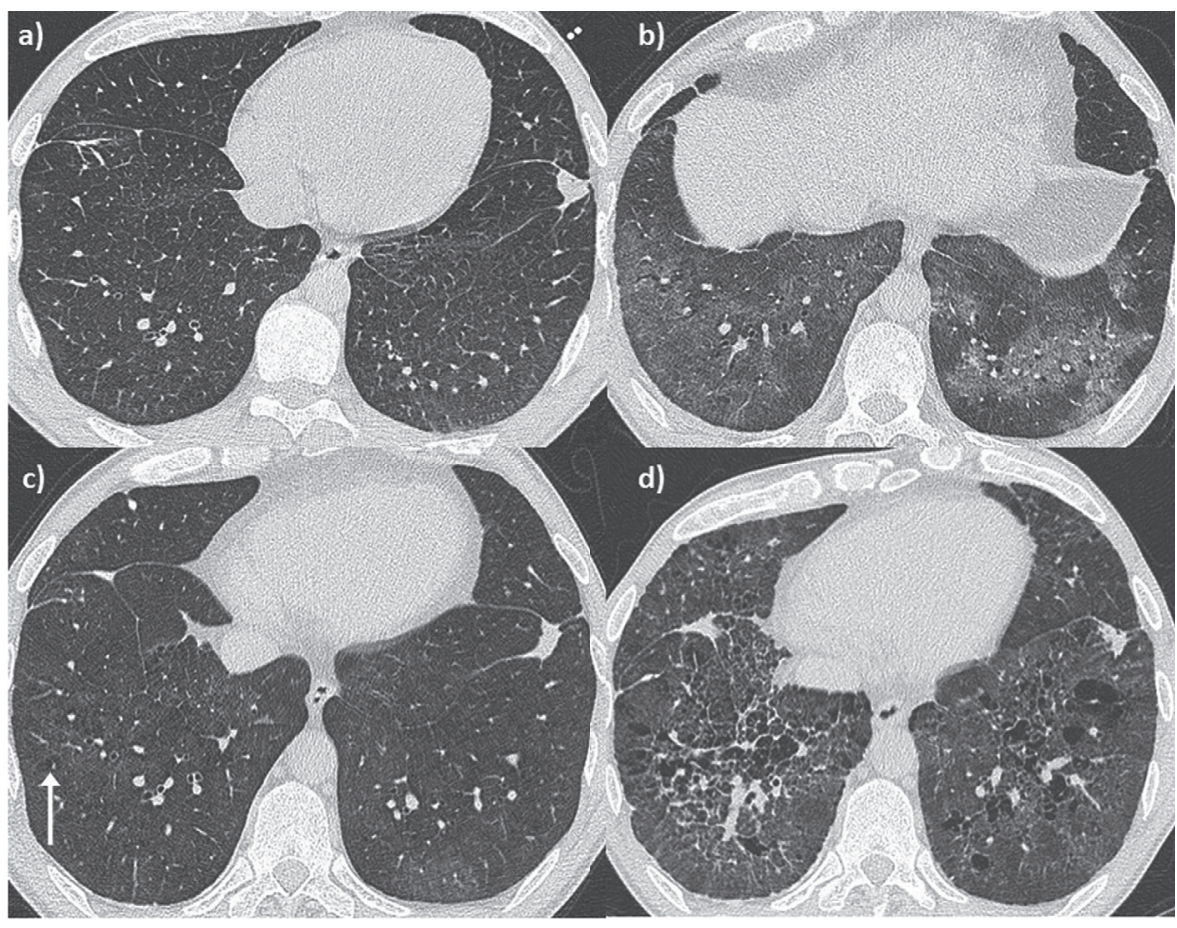

men seiner Tätigkeit Chemikalien versprühen und in Getreidespeichern arbeiten, wobei er jedoch stets eine Schutzmaske trug. Er war ehemaliger Raucher und hatte das Rauchen 10 Jahre vor der Manifestation seiner Probleme aufgegeben (5 Packungsjahre). Der Patient verneinte Symptome einer Kollagenose. Ein wiederholtes Autoimmun-Screening mit Untersuchung auf ANCA fiel bis auf eine leichte ANA-Positivität mit leicht gesprenkeltem Muster negativ aus. Die übrigen Bluttests zeigten eine mikrozytäre hypochrome Anämie und Positivität für Anti-Schilddrüsen-Antikörper. Während Anti-Transglutaminase-IgG-Antikörper gefunden wurden, konnte eine gastroenterologische Abklärung einschließlich von Duodenum-Biopsien eine Zöliakie ausschließen. Bei der BAL wurden mittels Perls-Färbung angefärbte pigmentierte Makrophagen beobachtet. Die Lungenfunktionsprüfungen ergaben normale Spirometrie-Werte und eine leichte Beeinträchtigung des Gasaustauschs (FEV 1 127\%; FVC 120,5\%; TLC 106\%; $\mathrm{T}_{\mathrm{LCO}} 84 \%$ und $\mathrm{K}_{\mathrm{CO}}$ 76\%). Bei dem Patienten wurde eine Behandlung mit niedrig-dosiertem Prednison begonnen, die zum Abklingen der Hämoptysen führte. Der Patient blieb mehrere Jahre lang funktionell und klinisch stabil, während die PrednisolonDosis langsam ausgeschlichen wurde. Sechs Jahre später beschrieb er erneut episodenhaft auftretende geringe Hämoptysen und Arthralgie, die auf kurze Behandlungszyklen mit Prednisolon in erhöhter Dosis ansprachen. Ein erneutes HRCT zeigte deutlichere Milchglasinfiltrate (Abb. 3b). Acht Jahre nach der Erstmanifestation wurden p-ANCA mit Anti-MPO-Positivität nachgewiesen $(14 \mathrm{U} / \mathrm{ml})$, was durch einen zweiten Test $(12 \mathrm{U} / \mathrm{ml})$ bestätigt wurde. Da der Patient weiterhin episodenhaft leichte Hämoptysen hatte, wurde der Hintergrundtherapie aus niedrigdosiertem Prednisolon Methotrexat hinzugefügt. Trotz relativ guter Kontrolle der Hämoptysen und nur gelegentlichen Episo- den, die durchgehend auf kurze Behandlungszyklen mit erhöhten Prednisolon-Dosen ansprachen, wurden 8 Jahre nach der Erstmanifestation im HRCT erste kleine Emphysembereiche sichtbar (Abb. 3c), die progressiv zunahmen und auf einem HRCT 2 Jahre später deutlicher imponierten. Wiederum wurde ein perivaskuläres Emphysem beobachtet (Abb. 3d). Parallel zu den Veränderungen im CT wurde eine progressive Verschlechterung des Gasaustauschs beobachtet. Bei im Wesentlichen stabilen SpirometrieWerten wurde ein $\mathrm{T}_{\mathrm{LCO}}$-Wert von $41 \%$ und ein $\mathrm{K}_{\mathrm{CO}}$-Wert von $42 \%$ gemessen, während die Echokardiographie einen Normalbefund zeigte.

\section{Patient 3}

Ein 16 Jahre alter junger Mann wurde mit der Diagnose einer IPH von der pädiatrischen Lungenabteilung in die ILD-Abteilung am RBH verlegt. Er hatte im Alter von 7 Jahren eine erste Hämoptysen-Episode gezeigt und litt seither unter rezidivierenden Episoden mit Hämoptysen, Atemproblemen und Husten. Vor dem Hintergrund der Symptome, rezidivierender Verschattungen in der Bildgebung (Abb. 5a) und einem negativen AutoimmunScreening wurde zunächst die Diagnose IPH gestellt. Ein interessanter Aspekt ist, dass im Alter von 6 Jahren eine HashimotoThyroiditis diagnostiziert worden war. Bei dem Patienten zeigte sich ein enger Zusammenhang zwischen Exazerbationen der IPH und Thyroiditis-Schüben. Bis zum Alter von 10 Jahren sprachen die Symptome und bildgebenden Befunde auf intermittierende Behandlungszyklen mit unterschiedlichen Dosen von oralen Kortikosteroiden an.

Im Alter von 11 Jahren wurde der Patient mit Hämoptysen und schwerer respiratorischer Insuffizienz in das örtliche Krankenhaus aufgenommen. Er wurde intubiert und auf die pädiatrische
104

Kompass Pneumol 2020;8:101-108 DOI: $10.1159 / 000505468$ 
Abb. 4. Patient 2. Histologischer Befund einer Lungenbiopsie. (a) Deutliche Akkumulation von hämosiderinbeladenen Makrophagen in den Alveolen (H\&E x 40). (b) Hämosiderinbeladene Alveolarmakrophagen und fokale perivaskuläre lymphoide Infiltrate (H\&E x 200). (c) Eiseninkrustation von Elastinfasern einer kleinen Arterie (H\&E x 200). (d) Kleines perivaskuläres nicht nekrotisierendes Granulom (H\&E X 200).

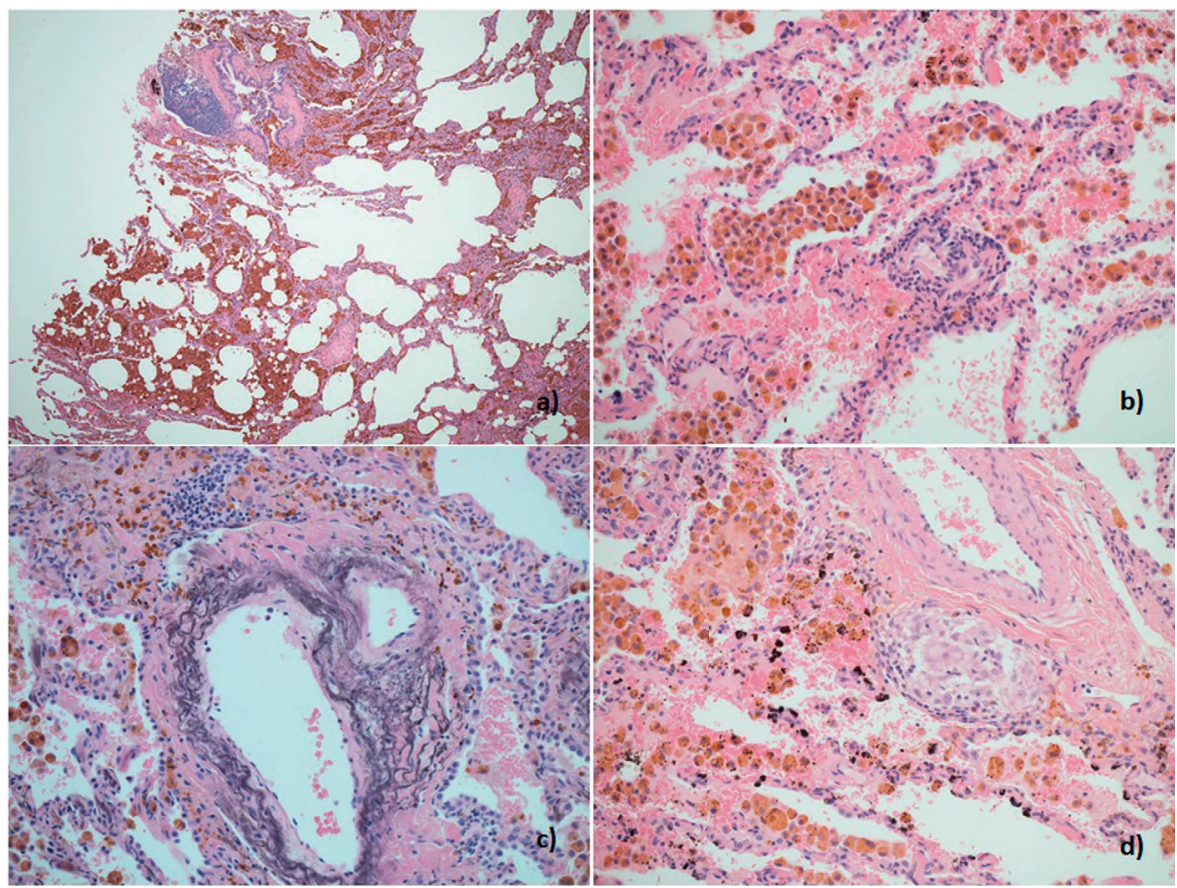

Abb. 5. Patient 3. Radiologischer Verlauf. Axiale CT-Bilder der Lungen. (a) Im Alter von 9 Jahren hatte der Patient einen akuten Schub und ein CT nach Kontrastmittelgabe zeigt knötchenförmige Milchglastrübungen, die auf späteren Röntgenaufnahmen des Thorax (nicht gezeigt) nicht mehr zu sehen waren. (b) Im Alter von 11 Jahren ist ein kavernenbildender Knoten im rechten Lungenunterlappen zu erkennen und um die Lungengefäße herum hat sich ein Emphysem ausgebildet (Pfeil). Der Knoten ist im Folge-CT (nicht gezeigt) nicht mehr zu sehen. (c) Im Alter von 15 Jahren hat das Emphysem stark zugenommen und wiederum ist eine perivaskuläre Komponente erkennbar. Zusätzlich ist ein akutes Milchglasinfiltrat zu sehen. (d) Im Alter von 20 Jahren sind polygonale Konsolidierungen im peripheren Lungenparenchym/der Pleura zu erkennen, die mit einer pleuroparenchymalen Fibroelastose vereinbar sind. Außerdem liegen ein Milchglasinfiltrat und Emphysem vor.

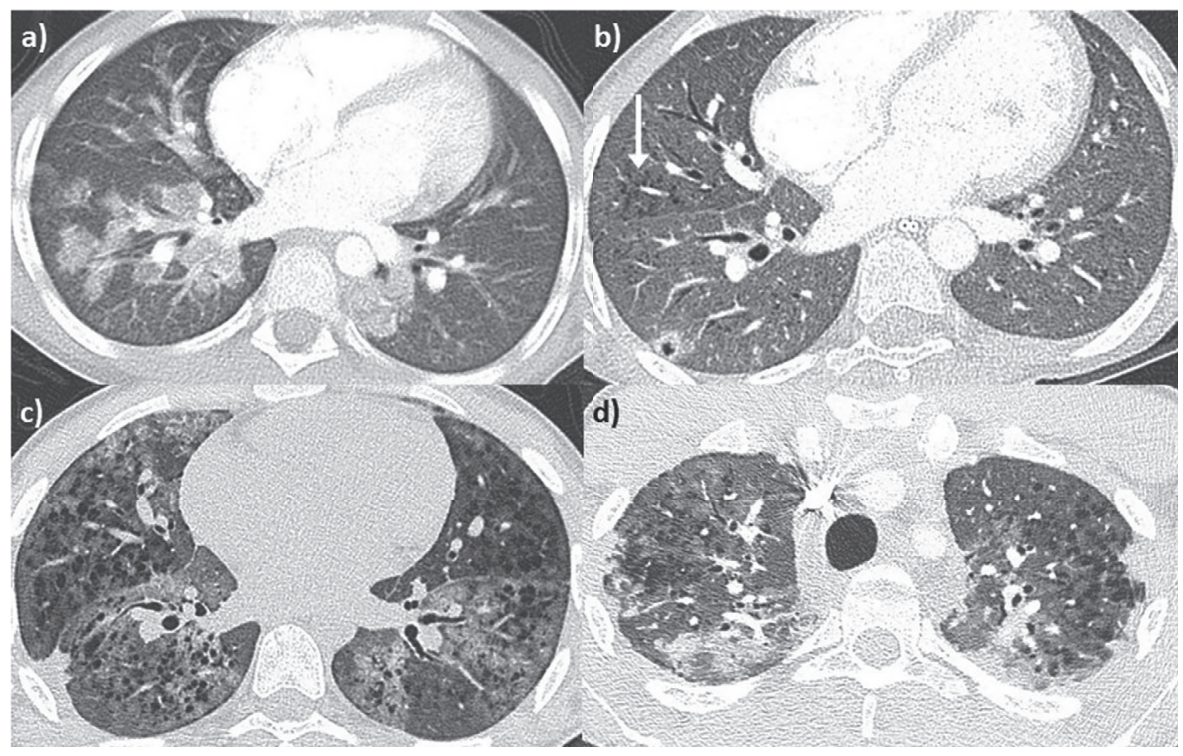

Intensivstation am RBH verlegt. Dort erfolgten eine BAL und chirurgische Lungenbiopsie. Die BAL ergab nach Perls-Färbung große Zahlen hämosiderinbeladener Makrophagen. Die Biopsie zeigte eine DAH ohne Anhalt für Vaskulitis (Abb. 6). Im HRCT waren zum Zeitpunkt der Biopsie diffuse Milchglastrübungen, ein perivaskuläres Emphysem und eine kleine Kaverne im rechten Unterlappen zu erkennen (Abb. 5b). Dies lenkte den Verdacht auf eine Vaskulitis, allerdings fielen wiederholte Tests auf ANCA negativ aus. Der Patient erhielt Antibiotika und intravenöse Kortikosteroide, worauf er gut ansprach. Bei der Folge-Bildgebung war die Kaverne nicht mehr zu sehen. Bei einer Spirometrie vor dieser akuten Episode hatte die $\mathrm{FEV}_{1} 41 \%$ und die FVC $37 \%$ betragen.
Bei der Entlassung wurde eine Erhaltungstherapie mit Hydroxychloroquin, niedrig-dosiertem Prednisolon und Azithromycin $250 \mathrm{mg}$ dreimal wöchentlich begonnen. Bei Schüben wurden kurze Zyklen von Prednisolon in erhöhten Dosen verabreicht. Ein Folge-HRCT 4 Jahre nach der Biopsie zeigte ein ausgedehntes Emphysem mit perivaskulärer Verteilung und überlagernden diffusen Milchglastrübungen (Abb. 5c). Der Patient hatte niemals geraucht und war niemals Passivrauchen ausgesetzt gewesen.

Bei seiner Aufnahme in die ILD-Klinik für Erwachsene und 8 Jahre nach der Biopsie, im Alter von 19 Jahren, wurde eine weitere Progredienz der radiologischen Befunde mit ausgedehntem Emphysem und einer pleuroparenchymalen Fibroelastose (PPFE) beider Oberlappen festgestellt (Abb. 6d). Die Lungenfunktion war 
Abb. 6. Patient 3. Histologischer Befund einer Lungenbiopsie. (a) Chirurgische Lungenbiopsie mit deutlicher Anhäufung pigmentierter Alveolarmakrophagen und verstreuten kleinen lymphoiden Aggregaten (H\&E x 100). (b) Alveolarmakrophagen mit grobkörnigem goldbraunem Pigment im Zytoplasma. Zu sehen sind eine reaktive Typ-2-Hyperplasie und leichte Erweiterung der Alveolenwände mit verstreuten kleinen Lymphozyten und minimaler Fibrose aber ohne Merkmale einer Kapillaritis oder Vaskulitis (H\&E x 200). (c) Die positive Reaktion des im Zytoplasma zu erkennenden Pigments in der Preußisch-Blau-Färbung nach Perls bestätigt, dass es sich um Hämosiderin handelt. Zu beachten auch die Eiseninkrustation von ElastinFasern in kleinen Gefäßen (Preußisch-Blau-Färbung nach Perls $\times 200$ ).

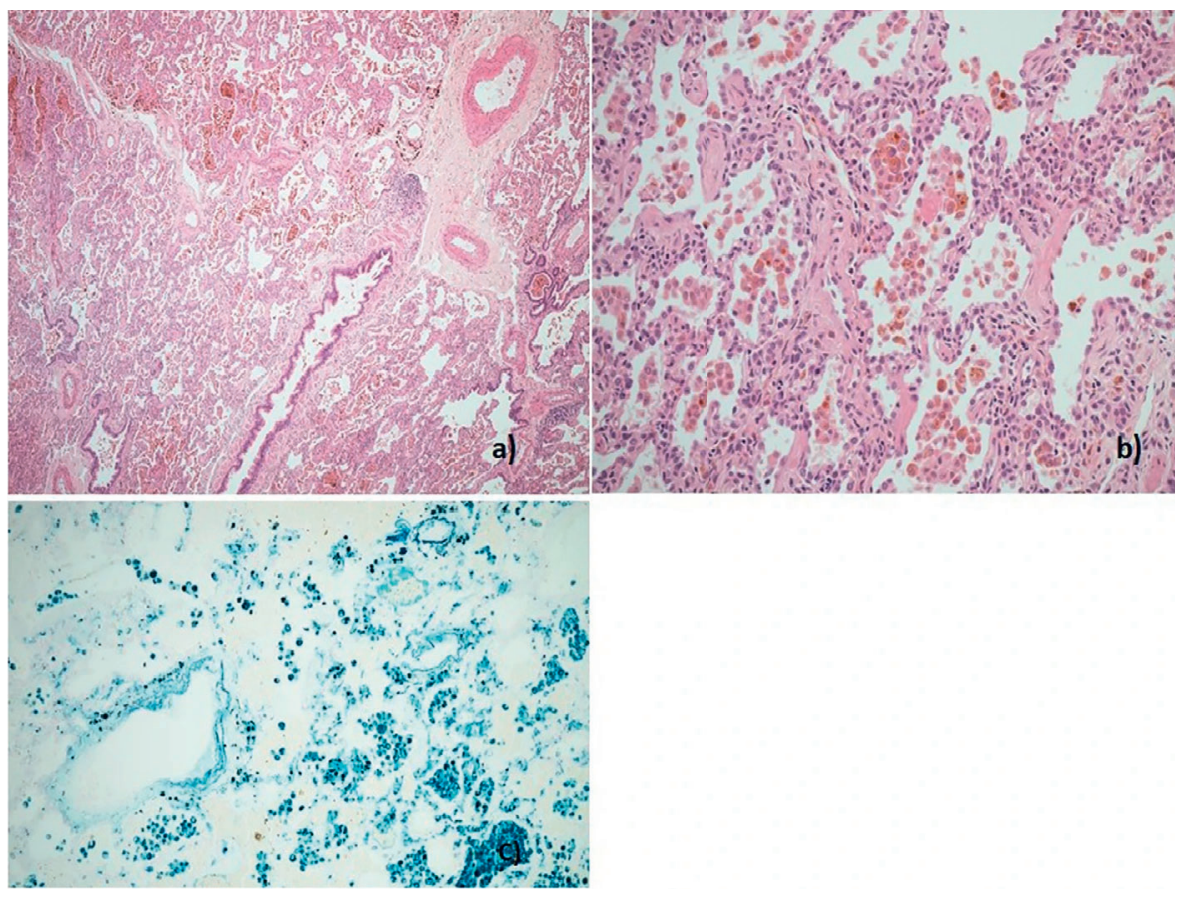

als Ausdruck des Emphysems in Kombination mit der PPFE stark eingeschränkt $\left(\mathrm{FEV}_{1} 54 \%\right.$, FVC 46\%, $\mathrm{FEV}_{1} / \mathrm{FVC} 99 \%$, $\mathrm{T}_{\mathrm{LCO}} 33 \%$ und $\mathrm{K}_{\mathrm{CO}} 68 \%$ ). Ein erneutes Autoimmun-Screening zeigte p-ANCA mit Anti-MPO-Positivität (4,6 U/ml). Angesichts dieser Ergebnisse wurde eine Behandlung mit niedrig-dosiertem Azathioprin begonnen, wobei Hydroxychloroquin und Azithromycin fortgesetzt wurden. Aktuell sind Symptome und Funktion stabil.

\section{Diskussion und Schlussfolgerungen}

Wir stellen hier 3 Patienten mit chronischer DAH vor, bei denen zunächst die Diagnose einer IPH gestellt worden war und die später MPO-ANCA-Positivität sowie im HRCT mit einem Emphysem vereinbare progrediente zystenartige Bereiche mit auffallender perivaskulärer Verteilung ausbildeten. Unserer Meinung nach sind diese 3 Fälle von Bedeutung, da sie erstens zeigen, dass mehrere Jahre nach Erstmanifestation einer IPH ANCA +/- eine Nierenbeteiligung auftreten können, und zweitens zeigen, dass sich aus den im Zusammenhang mit einer DAH auftretenden Veränderungen ein Emphysem entwickeln kann.

\section{Auftreten von MPO-ANCA-Antikörpern}

In verschiedenen Szenarien wurde ein Auftreten von spezifischen Antikörpern +/- anderen typischen extrapulmonalen Manifestationen eines Autimmun-Geschehens nach initialer pulmonaler Manifestation beschrieben $[3,4,5,6]$. Speziell im Hinblick auf das Auftreten einer MPO-ANCA-Positivität zeigten Längsschnittbeurteilungen bei Patienten, die initial eine idiopathische interstitielle Pneumonie gezeigt hatten, im Zuge der Verlaufsbeurteilung eine neu auftretende Anti-MPO- oder Anti-PR3-Positivität und/ oder offenkundige Vaskulitis [3].
Allerdings gibt es nur wenige Berichte mit detaillierten Angaben zu Serokonversionen für Autoimmunmarker (außer ANCA) nach initialer Diagnose einer IPH [7, 8]. Le Clainche et al. [4] berichten über 15 Kinder mit der Diagnose einer IPH, von denen nur eines bei einer späteren Untersuchung ANCA aufwies (keine Angaben zur Spezifität). Allerdings war bei den Kindern bei der Erstmanifestation kein Screening auf ANCA erfolgt, so dass keine Aussagen darüber gemacht werden können, ob es sich um eine echte Serokonversion handelte.

Eine nach vielen Jahren im Erwachsenenalter auftretende ANCAMPO-Serokonversion bei einem Patienten mit IPH wurde unseres Wissens nach nur in einem weiteren Fall beschrieben [5]. Allerdings handelte es sich bei der von Freitas et al. vorgestellten Patientin um eine 21 Jahre alte Frau, bei der im Alter von 4 Jahren eine IPH diagnostiziert worden war und die 12 Jahre nach der Erstmanifestation, im Alter von 16 Jahren, eine ANCA-Positivität ausbildete, die gut auf eine Immunsuppression ansprach. Darüber hinaus wurde nicht darüber berichtet, dass bei Verlaufsuntersuchungen emphysematöse Veränderungen zu sehen waren. Demgegenüber bildete sich die ANCA-Positivität bei den von uns beschriebenen Fällen im Erwachsenenalter aus, und alle Patienten benötigen weiter eine fortlaufende immunsuppressive Behandlung, um die DAH unter Kontrolle zu halten. Dies weist darauf hin, dass bei dieser seltenen Erkrankung Autoimmun-Phänomene eine Rolle spielen könnten, auch wenn initial eine «idiopathische» Form diagnostiziert wird. Diese Annahme wird auch durch die Beobachtung unterstützt, dass der zweite Patient Anti-Transglutaminase-IgA-Antikörper und eine AutoimmunThyroiditis und der dritte Patient eine Autoimmun-Thyroiditis hatte.
106

Kompass Pneumol 2020;8:101-108 DOI: $10.1159 / 000505468$ 


\section{Entwicklung des Emphysems}

Unsere Patienten bildeten im Verlauf zystenartige Verschattungen aus, die mit einem Emphysem vereinbar sind und bei der Erstvorstellung nicht zu sehen waren. Bei der ersten Patientin war erstmals 10 Jahre nach der Erstmanifestation ein begrenztes Emphysem zu sehen. Der zystenartige Bereich wurde in den folgenden 6 Jahren größer, während gleichzeitig isoliert der $\mathrm{T}_{\mathrm{LCO}} \mathrm{ab}$ nahm. Bei dem zweiten Patienten wurden erstmals 8 Jahre nach der Erstmanifestation im HRCT emphysematöse Veränderungen festgestellt, 20 Jahre nachdem er das Rauchen aufgegeben hatte. Nach weiteren 2 Jahren war das Emphysem in der radiologischen Bildgebung noch einmal signifikant fortgeschritten. Parallel dazu verschlechterte sich der Gasaustausch signifikant, während die Lungenvolumina normal blieben. Bei dem dritten Patienten war die IPH in der frühen Kindheit diagnostiziert worden, und ein Emphysem wurde erstmals 4 Jahre nach Diagnosestellung, im Alter von 11 Jahren, im HRCT sichtbar. Dieses verlief in der Folge progredient, obwohl der Patient weder aktiv rauchte, noch Passivrauchen ausgesetzt war.

Es ist zunehmend anerkannt, dass sich ein Emphysem im Zusammenhang mit interstitiellen Prozessen auch ohne relevante Raucheranamnese ausbilden kann. In einer Kohorte von lebenslangen Nichtrauchern mit einer ILD, die im Zusammenhang mit einer rheumatoiden Arthritis stand, hatten 27\% der Patienten ein Emphysem und auf eine obstruktive Ventilationsstörung hinweisende funktionelle Indizes [6], und von Patienten, deren ILD im Zusammenhang mit einer systemischen Sklerose stand und die außerdem ein Emphysem aufwiesen, waren 33\% lebenslange Nichtraucher [9]. In einer Kohorte von 233 Patienten mit Hypersensitivitätspneumonie, die auf eine Emphysembildung und PPFE untersucht wurden, waren 23\% der Patienten mit Emphysem lebenslange Nichtraucher [7].

Bei AAV wurde in einigen wenigen retrospektiven Fallserien und Fallberichten eine Emphysembildung beschrieben [8, 10, 11, 12]. In einer Kohorte von Patienten mit AAV, die 79 Patienten mit Granulomatose mit Polyangiitis (GPA) und 61 Patienten mit mikroskopischer Polyangiitis (MPA) umfasste, betrug die Gesamtinzidenz von emphysematösen Veränderungen $13 \%$ und zeigte keine Unterschiede zwischen den beiden Gruppen [12]. In der bislang größten veröffentlichten Serie beschrieben Yagamata et al. in einer Kohorte von Patienten mit MPA bei 37\% emphysematöse Veränderungen. Diese waren häufiger durch Bereiche mit geringer Abschwächung als durch zystenartige Läsionen gekennzeichnet. Allerdings stellten die Autoren nicht per se einen Zusammenhang zwischen den emphysematösen Veränderungen und einer alveolären Hämorrhagie her [13].

Die Pathogenese der Emphysembildung in Zusammenhang mit einer DAH und ANCA-Positivität ist unklar. Eine Möglichkeit ist, dass das in unseren 3 Fällen beobachtete Emphysem sekundär durch die in Kapillaren/kleinen Blutgefäßen auftretenden Entzündungsprozesse verursacht ist, wobei es durch Freisetzung schädlicher Substanzen wie proteolytischer Enzyme und freier Sauerstoffradikale zur Zerstörung der Alveolenwände kommt. Diese Hypothese wird durch die auffallende peribronchovasku- läre Verteilung des Emphysems unterstützt. Ein solcher Mechanismus wurde im Zusammenhang mit Kapillaritis [14] und hypokomplementämischer urtikarieller Vaskulitis, einer seltenen mit Immunkomplexen einhergehenden Vaskulitis der kleinen Blutgefäße, die durch niedrige Konzentrationen der Komplementkomponenten C1q und C4 gekennzeichnet ist, ins Gespräch gebracht. In seriellen histopathologischen Untersuchungen von Proben eines von Hunt et al. [15] beschriebenen Patienten war zu beobachten, dass sich die initialen im Zusammenhang mit der Kapillaritis auftretenden Veränderungen zu einem Emphysem mit obstruktiver Atemwegserkrankung entwickelten. Mehrere weitere Fallberichte zeigten diesen Zusammenhang im Kontext der hypokomplementämischen urtikariellen Vaskulitis [16, 17, 18, $19,20,21,22]$.

Eine weitere Möglichkeit ist, dass die ANCA-Antikörper selbst pathogen wirken. MPO-ANCA können Neutrophile aktivieren und zur Freisetzung von MPO, einem kationischen Protein, das von verschiedenen Immunzellen wie unter anderem Neutrophilen und Makrophagen exprimiert wird, führen, was eine Entzündungsreaktion zur Folge hat [23]. In einem Tiermodell des durch Rauchen induzierten Emphysems verhinderte ein Myeloperoxidase-Inhibitor das Fortschreiten der emphysematösen Veränderungen [24]. Eine Bedeutung von Antikörpern und insbesondere ANCA wird auch durch die Beobachtung unterstützt, dass Patienten mit ANA-Positivität signifikant häufiger eine Kombination aus pulmonaler Fibrose und Emphysem (CPFE) haben und einem höheren Anteil von MPO-ANCA-Positivität gegenüber Patienten mit idiopathischer pulmonaler Fibrose (IPF) ohne Emphysem. Darüber hinaus hatten CPFE-Patienten mit ANA- und/oder ANCA-Positivität erhöhte Zahlen von CD20+-Zellen, die im fibrotischen Interstitium in an Fibroblasten-Foci angrenzenden Bereichen lymphoide Follikel bildeten [25].

In den beiden ersten Fällen können wir nicht vollständig ausschließen, dass die zystenartigen Veränderungen und/oder das Emphysem mit dem früheren Zigarettenrauchen zusammenhingen. Sicherlich kann Rauchen zu dessen Entwicklung beigetragen haben. Allerdings hatte die erste Patientin eine wirklich zu vernachlässigende Raucheranamnese (1 Packungsjahr) und im Alter von 21 Jahren mit dem Rauchen aufgehört, also zu einem Zeitpunkt, als im HRCT keine zystenartigen/emphysematösen Veränderungen zu sehen waren. Der zweite Patient wies eine Raucheranamnese von 5 Packungsjahren auf, hatte das Rauchen aber aufgegeben und war seit 10 Jahren Nichtraucher, als das erste HRCT angefertigt wurde. Dieses zeige keinerlei emphysematöse Zerstörung. Der dritte Patient bildete in sehr jungen Jahren ein ausgedehntes Emphysem aus und wies keinerlei Exposition gegenüber Zigarettenrauch auf, was einen direkten Zusammenhang zwischen DAH, ANCA-Positivität und Emphysem unterstützt.

Es sind weitere Studien erforderlich, um die Beziehung zwischen DAH, ANCA-Positivität und Emphysembildung besser zu verstehen. ANCA-Positivität scheint bei Kindern mit IPH retrospektiv mit einem schlechteren Verlauf assoziiert zu sein [26]. Darüber hinaus zeigten Emphyseme, die im Zusammenhang mit Kollage- 
nosen und Hypersensitivitäts-Pneumonitis auftraten, eine schlechtere Prognose [6, 7, 9].

Die IPH ist eine seltene Erkrankung und es ist wenig über die Mechanismen bekannt, die an ihrem Auftreten beteiligt sind. Allerdings weisen der bekannte Zusammenhang mit der Zöliakie [27], das Ansprechen auf eine immunsuppressive Therapie und der mögliche Zusammenhang mit Autoimmun-Merkmalen [4, 28, 29] darauf hin, dass eine immunologische Überaktivität eine Rolle spielen könnte, selbst wenn keine eindeutige Autoimmunkrankheit nachgewiesen werden kann.

Bei diesen Patienten sollte auch bei zunächst idiopathischem Beginn immer eine immunsuppressive Therapie in Betracht gezogen werden. Patienten mit IPH sollten nicht nur bei der Erstmanifestation, sondern auch im Rahmen der Verlaufsbeobachtung auf ANCA gescreent werden. Dies gilt insbesondere für Patienten, die schlecht auf die Behandlung ansprechen oder bei denen der Verdacht auf eine Nierenbeteiligung besteht. Allerdings lohnt es sich darauf hinzuweisen, dass bei AAV nicht immer eine ANCAPositivität nachweisbar ist. Entsprechend ist der Nachweis von ANCA für die Diagnosesicherung oder den Beginn einer immunsuppressiven Therapie, sofern eine solche erforderlich ist, nicht notwendig [30]. Es sollten häufige umfassende Lungenfunktionsprüfungen einschließlich Bestimmung des $\mathrm{T}_{\mathrm{LCO}}$ erfolgen. Dies ist auch bei stabilen Spirometrie-Parametern wichtig, da es auch noch nach vielen Jahren durch emphysematöse Veränderungen zu einer subtilen Verschlechterung kommen kann. Dadurch lassen sich möglicherweise solche Fälle identifizieren, die von einer aggressiveren Therapie profitieren könnten, und gegebenenfalls Komplikationen im Zusammenhang mit derartigen emphysematösen Veränderungen verhindern.

\section{Beiträge der Autoren}

EAR und AS planten und schrieben den Fallbericht. AS erhob die Daten zu den Patienten. MK trug zur Datenerhebung und zum Schreiben des Fallberichts bei. EAR überwachte die Dateneingabe und prüfte das Manuskript. AR und AGN lieferten die histologische Befundung für die Patienten. AD und JLB lieferten die radiologische Befundung für die Patienten. JD lieferte die biochemische Befundung für die Patienten, insbesondere die Interpretation der ANCA-Ergebnisse. TC lieferte die nephrologische Befundung, insbesondere für Patientin 1. AUW prüfte und überarbeitete das Manuskript. Alle Autoren trugen zu der eingereichten Version des Manuskripts bei und genehmigten diese.

\section{Zustimmung zur Veröffentlichung}

Von allen 3 Patienten wurde die schriftliche Einwilligung nach Aufklärung eingeholt, ihre Patientendaten zu veröffentlichen. Alle Daten wurden retrospektiv zu einem Zeitpunkt erhoben, als die Patienten älter als 18 waren. Eine Einwilligung der Eltern/gesetzlichen Vertreter war nicht erforderlich.

\section{Disclosure Statement}

Die Autoren erklären, dass keine Interessenskonflikte bestehen.

\section{Literatur}

Die Literatur ist als Supplemental Material unter www.karger.com/ ?DOI=505468 abrufbar.

\section{Lizenzangabe}

Anna Stainer, Alex Rice, Anand Devaraj et al.: Diffuse alveolar haemorrhage associated with subsequent development of ANCA positivity and emphysema in three young adults. BMC Pulm Med 2019;19:185 (https:// doi.org/10.1186/s12890-019-0947-y), ${ }^{\circledR}$ The Author(s) 2019 (Übersetzung, Publisher's note, Verfügbarkeit der Daten und Materialien, Danksagungen, Finanzielle Unterstützung und Ethikerklärung gekürzt), lizensiert unter CC BY 4.0 (https://creativecommons.org/licenses/by/4.0/deed.de). 